\title{
La contradiction missionnaire. Discours et pratique des missionnaires méthodistes à l'égard des femmes africaines de Côte de l'Or (1835-1874)
}

\section{Anne Hugon}

\section{(2) OpenEdition}

Journals

Édition électronique

URL : https://journals.openedition.org/clio/374

DOI : $10.4000 /$ clio.374

ISSN : 1777-5299

Éditeur

Belin

Édition imprimée

Date de publication : 1 novembre 1997

ISBN : 2-85816-346-4

ISSN : $1252-7017$

\section{Référence électronique}

Anne Hugon, «La contradiction missionnaire. Discours et pratique des missionnaires méthodistes à l'égard des femmes africaines de Côte de l'Or (1835-1874) », Clio. Histoire, femmes et sociétés [En ligne], 6 | 1997, mis en ligne le 01 janvier 2005, consulté le 17 janvier 2023. URL : http:// journals.openedition.org/clio/374; DOI : https://doi.org/10.4000/clio.374

Ce document a été généré automatiquement le 17 janvier 2023.

Tous droits réservés 


\section{La contradiction missionnaire. Discours et pratique des} missionnaires méthodistes à l'égard des femmes africaines de Côte de l'Or (1835-1874)

\section{Anne Hugon}

1 À l'origine, la mission méthodiste ${ }^{1}$ en Côte de l'Or (ou Gold Coast, sud de l'actuel Ghana) paraît bien être exclusivement une affaire d'hommes: sollicité par un groupe d'Africains chrétiens qui souhaitent approfondir leur connaissance des Écritures et parmi lesquels ne figure pas une seule femme, le premier missionnaire méthodiste qui pose pied à Cape Coast en 1835 est un homme. Il faut attendre 1894 pour voir arriver dans la région la première femme missionnaire, Miss Amelia Jackman. D'autre part, quoique les missionnaires hommes soient encouragés à partir mariés, ils ne sont pas très nombreux à le faire (seize sur trente-neuf, entre 1835 et 1874) et les épouses sont souvent contraintes de rentrer rapidement en métropole pour raisons de santé, lorsqu'elles ne meurent pas dans les premiers mois. De ce fait, le nombre d'Européennes liées à la mission demeure faible durant ces quarante premières années.

2 Néanmoins, les missionnaires vont être tenus de se pencher rapidement sur la question des femmes, ne serait-ce qu'en raison d'impératifs pratiques: comment prétendre évangéliser si l'on exclut de la prédication une moitié de la population? Cet article se propose donc d'explorer la place des Africaines dans une mission britannique en Gold Coast vers le milieu du XIXe siècle, avant l'annexion de cette région par la GrandeBretagne en 1874.

3 La Côte de l'Or est alors une région où les contacts entre Européens et Africains ${ }^{2}$ remontent à plus de trois siècles: les phénomènes d'interaction et de modifications culturelles n'y sont pas nouveaux mais le christianisme, dans ses versions catholique puis protestantes, n'a pas réussi à gagner beaucoup d'adeptes ${ }^{3}$. Les rares chrétiens 
autochtones (quelques dizaines tout au plus vers 1830) font généralement partie de l'élite marchande. Sur les plans culturel, matrimonial et économique, ces grandes familles africaines sont fort proches des Européens qui vivent et commercent dans les forts jalonnant la côte - Danois, Néerlandais et Britanniques, ces derniers finissant par évincer les autres entre 1840 et 1874 . Enfin, depuis le début du XIXe siècle, la Côte de l'Or connaît également, du point de vue économique, une reconversion, lente mais plutôt réussie, de la traite négrière vers le commerce d'huile de palme.

C'est donc dans un contexte d'importantes mutations économiques, politiques et culturelles que s'implante en 1835 une mission protestante britannique, qui va à son tour accélérer certains de ces changements. A partir des archives de la $\mathrm{WMMS}^{4}$, il s'agira de comprendre quelle place les missionnaires ${ }^{5}$ voulaient réserver aux femmes africaines, à la fois au sein de l'institution méthodiste et dans la société chrétienne qu'ils appelaient de leurs voeux; puis de mesurer les effets pratiques, pour le moins ambigus, de leur programme.

De l'infériorité sociale des femmes africaines

Cependant, avant d'aborder l'analyse du discours et de la pratique missionnaires, il convient de comprendre quels étaient le statut et la place des femmes dans la société côtière ${ }^{6}$ au moment où les premiers missionnaires s'y sont implantés. La plupart des sources européennes de l'époque présentent la situation des femmes en termes d'infériorité sociale - et comme on le verra, les missionnaires ne font pas exception à cette règle. Cependant, l'utilisation de ces sources est toujours délicate. D'une part, le relativisme culturel n'étant pas le fort des Occidentaux du XIXe siècle, leur vision est largement brouillée par le modèle européen, qui sert de référence constante... mais toujours implicite. D'autre part, les jugements de valeur sont si intimement liés à leur description des sociétés africaines qu'il est parfois difficile de démêler l'exposé de la dénonciation. En outre, avec une fâcheuse tendance à la généralisation, ces auteurs négligent généralement la diversité des sociétés africaines: en l'occurrence, leurs remarques sur «la femme africaine " rendent mal compte de situations en fait très variées. Enfin et surtout, certaines observations dues à des Européens sont contredites par des données sociales, économiques ou culturelles auxquelles ils se montraient peu sensibles - voire tout-à-fait imperméables.

7 Il faut donc réexaminer la place des femmes à différents niveaux de la société côtière, pour voir ce qu'il en est de leur supposée infériorité sociale. Tout d'abord, sur le plan socio-culturel, la société fante est matrilinéaire: les membres d'un même lignage (abusua) se reconnaissent tous une ancêtre commune. Le système de parenté confère donc aux femmes une place très importante. Mais cette place demeure à bien des égards purement symbolique, sans conséquence tangible dans l'organisation politique ou économique. Ainsi, l'héritage ne passe-t-il pas par les femmes elles-mêmes mais par leurs frères : fonctions politiques et biens matériels se transmettent de l'oncle maternel au neveu. Le système de parenté matrilinéaire ne garantit donc pas automatiquement aux femmes une place plus enviable que dans des sociétés patrilinéaires contrairement à l'idée répandue qui repose sans doute sur une confusion entre l'ordre symbolique et celui de la réalité?.

8 Si l'on peut parler d'égalité des droits dans la société civile (les femmes pouvant ester en justice, par exemple), elles demeurent subordonnées à leur époux dans les relations matrimoniales. Quant aux fonctions de prestige, politiques ou sociales, elles en sont presque toujours exclues, de fait sinon de droit: à peine relève-t-on, 
exceptionnellement, la présence d'une femme à la tête d'une compagnie militaire ${ }^{8}$. De surcroît, la société fante connaît une forte division sexuelle du travail, qui n'est pas particulièrement favorable aux femmes. C'est à elles qu'est dévolu le travail domestique, d'autant moins prestigieux que la notion même de vie domestique n'est pas très valorisée. C'est aux femmes encore que revient l'éducation des enfants (garçons et filles), du moins jusque vers sept ou huit ans, âge auquel les enfants de sexe masculin sont confiés à leur père, dont ils apprennent le métier. Les professions réservées aux femmes sont généralement considérées comme mineures, y compris lorsqu'elles sont indispensables économiquement (orpaillage, poterie, commerce local).

9 Malgré ces restrictions, les femmes de Côte de l'Or jouissent dans le domaine économique d'un avantage majeur: leur indépendance. En effet, même mariées, elles peuvent user librement du produit de leur travail, qui n'est pas soumis au contrôle de leur mari ${ }^{9}$. Cette pratique est particulièrement favorable aux (nombreuses) commerçantes, qu'il s'agisse de la simple vendeuse de «kenke $»^{10}$ ou des quelques femmes de l'élite qui dirigent de florissantes entreprises commerciales au milieu du XIXe siècle ${ }^{11}$.

10 Une historienne actuelle, l'une des rares à se pencher sur la question du statut des femmes et des rapports sociaux entre hommes et femmes, conclut pour sa part : « En dernière analyse, le statut d'une femme dans la société était déterminé par les hommes (male-determined). Si son mari était riche ou puissant, ou si les hommes de son lignage occupaient un rang important, son statut s'en trouvait rehaussé d'autant. À l'inverse, il semble qu'il ait été extrêmement difficile, voire impossible, pour une femme, d'atteindre une position plus élevée que celle des hommes auxquels elle était liée. ${ }^{12} \mathrm{En}$ somme, le problème de la situation des femmes en Côte de l'Or tient moins à un statut inférieur qu'à un statut non-autonome - deux notions qui, bien qu'elles se recouvrent partiellement, ne sont pas pour autant synonymes. À partir de cette analyse, deux questions se posent : pourquoi les Européens ont-ils analysé la situation des femmes en termes d'infériorité plutôt que de dépendance ? Et pourquoi ont-ils négligé une donnée pourtant fondamentale, l'autonomie économique des femmes?

11 En fait, cette dernière omission ne relève pas du hasard: le discours européen sur le statut des femmes s'appuie sur des considérations morales beaucoup plus que sur des observations matérielles ${ }^{13}$. Et davantage encore que les auteurs laïcs, les missionnaires vont se montrer sensibles aux questions éthiques. Ainsi, l'une des constantes de leurs réflexions sur les femmes réside-t-elle dans cette notion : la déchéance morale, déclinée sous plusieurs vocables. Le directeur de la mission de Gold Coast écrivait par exemple en 1838 : « Je suis au regret de dire que les femmes de Cape Coast sont dans un réel état de dépravation $»^{14}$. Certes, elles ne sont pas seules soumises à ce jugement mais dans le discours général sur la déchéance des Africains, les femmes occupent, si l'on ose dire, une place privilégiée - le bas de l'échelle de la dépravation...

12 Comment expliquer cette différence de traitement? À la fois par des considérations très générales sur la place des femmes dans l'imaginaire chrétien, que l'on ne saurait développer ici, et par des facteurs propres à la Gold Coast. Parmi eux, la question de la " nudité » obsède littéralement les missionnaires ${ }^{15}$, bien qu'il s'agisse en fait d'une semi-nudité : on ne se couvre pas le buste. Pour un missionnaire méthodiste, passe encore que les hommes aillent « presque nus »; mais que les femmes ne se couvrent pas la poitrine, voilà une preuve irréfutable de leur déchéance morale. 
13 Les missionnaires achèvent de s'en convaincre en assistant à des scènes de danse, qui représentent pour eux l'illustration de la licence qui régnerait chez les Fante. Bien que les hommes y participent également, c'est, une fois encore, l'attitude des femmes qui choque le plus les méthodistes. L'un d'eux dénonce violemment « les danses de femmes, les danses ineptes de femmes ${ }^{16}$, tandis que son successeur affirme : «Pour les femmes, le summum du bonheur est de danser au son du tambour, le visage couvert de peinture, avec de grands gestes frénétiques ${ }^{17}$. Ce qui est en cause ici, c'est une gestuelle et un comportement qui semblent à l'opposé de la retenue et de la décence dévolues aux femmes dans la culture victorienne. La raison pour laquelle les missionnaires jettent ainsi l'opprobre sur les Africaines de Gold Coast est qu'elles manifestent une gestion du corps différente de celle qui prévaut alors dans la pudibonde Angleterre. Les wesleyens ne voient dans les danses qu'une combinaison regrettable de plaisir et de postures déplacées, qui leur semblent symptomatiques de la pire débauche.

14 Ainsi, lorsque les missionnaires dénoncent la dépravation des sociétés côtières, ils accablent tout particulièrement les femmes. Pourtant, paradoxalement, celles-ci bénéficient d'une absolution partielle, qui ne s'applique pas aux hommes : globalement, les femmes sont considérées comme moins condamnables pour les fautes qu'elles commettent, car elles seraient victimes d'un système à la fois social et éthique dont elles ne peuvent être tenues pour responsables. La clé de voûte de ce système serait

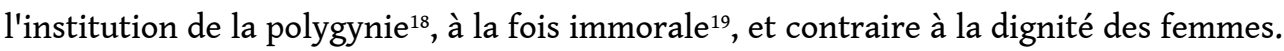
La polygynie est considérée comme un système entièrement subi par les Africaines, qui en sont à la fois "dupes et victimes $»^{20}$. S'il se trouve des femmes pour la défendre ${ }^{21}$, leurs arguments sont balayés d'un revers de main, les missionnaires invoquant un phénomène d'aliénation : «Les pauvres créatures elles-mêmes tiennent sur le sujet les raisonnements auxquels on peut s'attendre de la part de pauvres païennes ignorantes ; et elles concluent qu'il vaut mieux pour elles avoir une maison confortable et être entretenues que de vivre honnêtement dans la pénurie et le besoin. $»^{22}$

Aux yeux des méthodistes britanniques, l'infériorité sociale des Africaines doit être imputée à la polygynie, dans la mesure où celle-ci prive les femmes d'exercer en toute souveraineté leur rôle domestique. Freeman, directeur de la mission de Gold Coast, déclare ainsi dans un rapport à ses employeurs que «la pauvre femme n'a presque aucun contrôle sur sa propre maison », avant d'ajouter : "En entrant dans n'importe quelle maison indigène, on remarque que Mrs. A. ou B. est considérée comme un être inférieur. ${ }^{23}$ Le statut domestique des femmes est la preuve de leur infériorité. En outre, la polygynie est dénoncée par les missionnaires parce qu'elle entraîne la subordination des Africaines: «La femme païenne se considère comme une simple créature à l'usage de l'homme et elle inculque à sa fille cette seule et unique idée. $»^{24}$

16 Freeman ne se contente pas de mettre en cause cette conviction: il se propose d'y remédier en promouvant un autre système, dérogeant d'ailleurs à l'obligation méthodiste de ne s'occuper que du domaine spirituel ${ }^{25}$. L'un des buts proclamés consiste à améliorer la condition des femmes, et Freeman trouve des accents émancipateurs lorsqu'il déclare vouloir «les élever à la place qui leur revient dans l'échelle des êtres $»{ }^{26}$ Reste à savoir en quoi consiste cette place, et quelles mesures prennent les missionnaires pour mener à bien un programme qui se prétend résolument progressiste.

La pratique méthodiste et ses effets sur la situation des femmes 
$18 \mathrm{Au}$ XIXe siècle, l'influence des missionnaires sur la société côtière est encore assez réduite: ils ne sont jamais plus d'une demi-douzaine sur le terrain, et malgré la technique de la prédication en plein air, l'Église ne compte vers 1875 que quelques milliers d'adhérents. Néanmoins, les wesleyens ont à leur disposition un moyen efficace pour agir sur les plans culturel et social : l'école. Leurs établissements scolaires sont en effet largement fréquentés par des enfants de familles non chrétiennes, sensibles aux promesses de promotion sociale qu'offre la maîtrise de la lecture et de l'écriture. Mais l'enseignement méthodiste ne se limite pas à ces aspects techniques : les missionnaires, qui voient dans l'école la pépinière de l'Église, la mettent à profit pour inculquer aux enfants de nouvelles valeurs. Ainsi, l'examen du programme scolaire destiné aux filles montre bien quelle place, à terme, ils réservaient aux femmes dans la société africaine christianisée.

Il faut d'abord préciser qu'à l'exception de Cape Coast, capitale régionale, les wesleyens ne disposent pas d'écoles de filles ${ }^{27}$, du moins plus après les années 1840. Sur la vingtaine d'écoles méthodistes que compte la Gold Coast vers 1870 (dont un bon nombre de très modestes écoles rurales), on ne trouve que des écoles « mixtes »-c'està-dire, en l'occurrence, des établissements fréquentés par une écrasante majorité de garçons et par une proportion de filles allant de... $0 \%$ à $20 \%$ selon les cas ${ }^{28}$. La faiblesse de ces chiffres s'explique - rien d'original à cela - par les réticences des parents à scolariser leurs filles, réticences que les missionnaires imputent précisément au statut social des femmes: "Nous avons ouvert une école pour filles le 17 mai. Au préalable, l'auteur de la présente avait parcouru toute la ville mais avec un résultat décevant : on ne nous avait promis que deux fillettes. Ici, le sexe féminin est tellement dégradé, considéré comme si inférieur au sexe masculin, que l'idée même qu'on veuille les instruire était l'objet de dérision et de moqueries - comme si c'était là l'idée la plus absurde jamais conçue par l'esprit humain. $»^{29} \mathrm{Si} \mathrm{l'on} \mathrm{en} \mathrm{croit} \mathrm{certains} \mathrm{témoignages} \mathrm{de}$ méthodistes, les mères semblent s'opposer plus que les pères à la scolarisation des enfants (garçons ou filles) ${ }^{30}$, ressenties ans doute comme une concurrence à leur rôle d'éducatrices.

20 En dépit de ces objections, les missionnaires parviennent parfois à obtenir suffisamment d'écolières pour leur concocter un programme d'études spécifique, procédure qui en elle-même montre assez qu'ils n'ont nullement l'intention d'éduquer indistinctement garçons et filles ${ }^{31}$. Après des matinées vouées aux disciplines générales ${ }^{32}$, chaque après-midi est consacrée dans son intégralité aux travaux manuels, avec une prédominance absolue des cours de couture. Les travaux d'aiguille occupent donc un mi-temps ${ }^{33}$, aux dépens des matières intellectuelles, lesquelles, en revanche, absorbent les garçons à plein temps.

21 Cette insistance sur la couture correspond à plusieurs soucis des missionnaires. D'une part, ils espèrent ainsi modifier les habitudes vestimentaires des femmes, comme en témoigne ce wesleyen après avoir rendu visite à des écolières : «Chacune était habillée très joliment à la mode européenne ; voici seulement quelques années, ces filles avec qui je prenais le thé couraient à travers les rues, presque nues. Mais grâce à l'instruction qu'elles ont reçue à l'école, elles savent coudre, lire et écrire et arborent une apparence très différente. $~^{34} \mathrm{D}$ 'autre part, les travaux d'aiguille ne constituent que la partie visible d'un projet plus vaste, consistant à faire des petites Africaines des maitresses de maison accomplies. En effet, on se rappelle que Freeman déplorait le faible contrôle exercé par les femmes sur leur foyer ${ }^{35}$ : quand il se proposait de «les 
élever à la place qui leur revient dans l'échelle des êtres ", il s'agissait en réalité de les former aux travaux de la maison. Il insiste abondamment sur ce point, ainsi lorsqu'il écrit : «Quand je marie deux de nos adhérents, j'en profite pour attirer leur attention sur l'économie domestique. J'estime qu'ils ont grand besoin d'information sur ce chapitre. Si ma chère épouse avait été épargnée, elle aurait certainement été très utile aux femmes de Cape Coast. Bon nombre d'entre elles la sollicitaient sans arrêt pour qu'elle les instruise dans les affaires domestiques. $»^{36}$

Le programme méthodiste consiste donc à faire "retrouver " aux Africaines leur vraie place, à savoir la maison - projet qui se décompose en trois volets : bonnes maîtresses de maison, mais aussi bonnes épouses (dans le cadre d'un mariage monogame) et bonnes mères. Instruire les filles ne saurait cependant constituer un but en soi, car si Freeman affirme qu'une "école de bon niveau est aussi nécessaire pour les filles que pour les garçons ", c'est pour ajouter aussitôt : "En effet, est-il normal d'obliger de jeunes hommes instruits à prendre pour épouses des femmes tout juste assez bonnes pour des pêcheurs païens? ». Ainsi l'école sert-elle essentiellement à former les futures épouses chrétiennes des adhérents de l'Église wesleyenne ${ }^{37}$. Quant à en faire de bonnes mères, il s'agit aussi d'une priorité, puisque Freeman estimait que les femmes nonchrétiennes se montraient "plus ou moins apathiques concernant le bien-être de leur enfant $»^{38}$. À l'inverse, dans un questionnaire adressé par les responsables londoniens de la WMMS sur les couples chrétiens, à la question «Les mères s'occupent-elles bien de leurs enfants?", il répondait par l'affirmative ${ }^{39}$. Le modèle proposé par les méthodistes est donc clairement celui d'une famille nucléaire, tournant autour d'un personnage féminin dont l'attention et l'énergie sont entièrement absorbées par la maison, l'époux et les enfants.

Largement inspiré du modèle bourgeois européen, ce projet n'a rien d'émancipateur et ne présente guère d'avantages immédiats pour les Africaines. Car en promouvant ce système, les missionnaires leur ôtent l'un de leurs principaux atouts dans la société traditionnelle: leur indépendance économique. Confinées à l'espace domestique, les femmes ne sont plus censées exercer d'activités extérieures rémunératrices. C'est aux hommes de pourvoir aux besoins de la famille, comme le montre la question posée à Freeman par ses supérieurs: "Les maris [chrétiens] obligent-ils leur femmes à travailler pour leur entretien? $»^{40}$. Le fait même que la question soit posée en termes «d'obligation » indique bien que pour les wesleyens, la réponse devrait être « Non » et d'ailleurs, Freeman répond effectivement par la négative.

Exclues de la sphère économique "active ", les épouses se retrouvent en situation de dépendance accrue. Plus vulnérables dans ce nouveau système, elles ne peuvent compter que sur la bonne volonté de leur mari ${ }^{41}$ et n'ont guère de recours dans le cas contraire. Il est vrai qu'autour de l'Église méthodiste se sont constitués des réseaux d'entraide, qui remplacent les solidarités traditionnelles. Fonctionnant bien lorsqu'il s'agit de soutenir une petite communauté wesleyenne en conflit avec les «païens »du même village, ils pourraient également servir en cas de difficultés au sein d'une famille chrétienne.

L'un des problèmes auxquels les Africaines de Côte de l'Or sont régulièrement confrontées est celui de la violence domestique, qui n'épargne pas les foyers méthodistes. Or les sources de la mission montrent que les femmes se tournent parfois vers les responsables méthodistes pour trouver un appui : «A midi, rapporte le journal de Thackwray en date du 19 mars 1841, est survenu un incident qui nous a causé bien 
du tourment : il s'agissait d'un homme qui battait sa femme et en plus, c'est un membre de notre Église. Mr. Hesk est parti voir de quoi il retournait et je l'ai accompagné. Nous avons trouvé la femme en pleurs, elle saignait du nez et avait les yeux au beurre noir. Ce n'était pas une de nos adhérentes mais une pauvre païenne ignorante, venue de l'intérieur, que cet homme, avant de se convertir, avait accepté de prendre pour femme à l'essai, et de garder si elle lui convenait. Il semble que récemment, il ait prévu de l'épouser à la manière chrétienne mais malheureusement est apparu un désaccord. La femme, qui semble-t-il n'était pas sans torts, a menacé de le quitter et de retourner chez elle en brousse ; il l'a frappée, causant ainsi un grand émoi. $»^{42}$ Le fait que deux missionnaires se déplacent pour cette affaire semble démontrer que la violence conjugale dans un couple chrétien n'est pas prise à la légère. Cependant, la conclusion de cet incident est loin de corroborer cette impression : «Mr. Hesk s'est fait relater les faits, a prononcé un petit discours sur les devoirs conjugaux et ordonné au mari de venir le voir le soir même, accompagné de son chef de classe ${ }^{43}$. Là, devant une assemblée des chefs de classe, le cas a été examiné du point de vue ecclésiastique. Et l'homme, qui paraissait humble et repentant, a été reconnu coupable de faute grave. Mais on l'a recommandé à l'indulgence de ses pairs au motif que les leaders ayant eux-

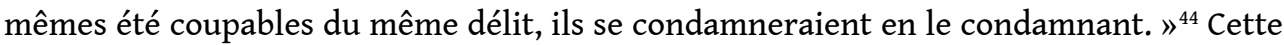
dernière phrase en dit long à la fois sur la fréquence des cas de violence domestique et sur l'incapacité de l'Église à venir en aide aux adhérentes qui en auraient besoin. De fait, comme le montre un autre document, il ne s'agit nullement d'une question prioritaire pour les missionnaires, qui ne s'en émeuvent guère. Un père de famille ayant battu sa femme qui refusait d'envoyer les enfants à l'école, l'auteur conclut : « Si condamnable que soit ce mode de correction, il a fait grande impression sur les autres mères et leur opposition a immédiatement cédé. $»^{45}$

En somme, les efforts que consentent les wesleyens pour l'amélioration de la condition des Africaines sont subordonnés à des impératifs supérieurs, tels que la promotion d'un nouveau modèle de famille et la diffusion du christianisme sur cette base familiale. À cet égard, ils se situent dans la lignée la plus conservatrice de Wesley, qui préconisait de remédier aux problèmes sociaux par des exercices spirituels.

Une Église peu féminisée

Cette incapacité des missionnaires à répondre aux problèmes spécifiques des femmes (chrétiennes ou non) ${ }^{46}$, explique probablement en partie l'une des caractéristiques sociales de l'Église méthodiste d'alors en Côte de l'Or: la faiblesse de ses effectifs féminins. Ce trait est d'autant plus frappant qu'en Grande-Bretagne, la même Église est très largement féminine : «Le méthodisme est en quelque sorte une branche féminine du protestantisme anglais. Les méthodistes ont toujours appartenu en majorité au sexe faible $»^{47}$. Ceci s'explique précisément par le fait que les "vices» dénoncés par les wesleyens rejoignent certaines préoccupations des femmes, qui luttent en particulier contre la brutalité et l'alcoolisme.

En Côte de l'Or, la faiblesse numérique des femmes dans l'Église méthodiste est en fait inégale selon les niveaux de la hiérarchie. Si l'on observe des registres de baptême des circuits $^{48}$ de Cape Coast et Anomabu, on dénombre en moyenne, sur trente ou quarante ans, une proportion de $40 \%$ de femmes et fillettes sur l'ensemble des baptisés ${ }^{49}$. On peut donc conclure à une sous-représentation réelle, mais pas excessive, du sexe féminin dans le groupe des baptisés. Pour ce qui est non plus des baptisés mais des adhérents à l'Église, on ne dispose malheureusement pas de chiffres permettant 
d'établir le pourcentage de femmes ${ }^{50}$. En revanche, la correspondance permet de conclure à une sous-représentation, peut-être plus nette encore si l'on en croit les missionnaires : "Il y a une bien plus grande proportion d'hommes que de femmes " déplorait Freeman en 1838, pour renchérir quelques années plus tard, cette fois au sujet d'un circuit particulier : «la proportion de femmes est très faible » ${ }^{51}$.

Cette tendance décline peut-être avec les années, puisque les responsables méthodistes n'en font plus état, mais parmi les responsables de l'Église, les femmes demeurent très minoritaires. Ainsi, elles brillent par leur absence au plus haut niveau puisqu'on ne compte aucune femme missionnaire africaine ${ }^{52}$. Au niveau intermédiaire, celui des «cadres » de l'Église, elles sont encore peu nombreuses: en 1842, on ne trouve que deux class leaders féminines pour seize class leaders masculins dans le circuit de Cape Coast (et une pour quinze dans celui d'Anomabu). En 1863, elles n'y sont encore que quatorze à remplir cette fonction contre trente-sept hommes. Et encore, le circuit de Cape Coast n'est-il guère représentatif de l'ensemble, puisqu'ailleurs, les proportions sont toujours de l'ordre d'une femme pour dix hommes. Enfin, même dans le corps enseignant, les femmes sont rares. Dans les écoles mixtes, on n'emploie que des maitres, ce qui signifie que les institutrices ne pratiquent que dans quelques écoles de filles. En 1865, on ne recense que cinq femmes pour trente-deux hommes parmi les enseignants des établissements wesleyens.

Les femmes cumulent donc deux faiblesses dans l'Église méthodiste : l'une quantitative (elles y sont globalement sous-représentées) et l'autre qualitative (elles sont nettement sous-représentées aux niveaux les plus hauts). A cela s'ajoute une donnée économique qui ne fait que renforcer ce trait: les salaires des institutrices recrutées par les missionnaires sont sensiblement inférieurs à ceux des maîtres d'école. Prenons l'exemple de Cape Coast, qui abrite la plus grande école méthodiste. En 1867, le maître d'école et son assistant gagnent respectivement 50 et 40 livres sterling par an. Dans l'école de filles, l'institutrice reçoit pour sa part 40 livres et son assistante 8 livres et 2 shillings. Cette disparité se retrouve dans d'autres localités et à d'autres moments : en 1869, à Accra, le maître est payé 24 livres annuelles tandis que la maîtresse (qui n'est autre que l'épouse du premier) perçoit 13 livres. Certes, ces traitements correspondaient à des critères précis prenant en compte le degré de qualification de l'employé-e $\mathrm{e}^{53}$. Mais le fait que dans l'ensemble, les femmes soient moins bien payées parce que moins qualifiées, reste révélateur : en effet, la plupart d'entre elles ont reçu leur formation... dans des établissements méthodistes !

En définitive, apparaît clairement une contradiction entre les objectifs affichés par les missionnaires et leur pratique. Leur analyse de la situation des femmes en Côte de l'Or les amène à vouloir améliorer leur condition par divers moyens tels que l'instruction ou le mariage monogame. Mais plus globalement, leur but consiste à développer le modèle de la famille occidentale du XIXe siècle, qui leur parait garantir aux femmes un sort préférable à celui des Africaines de Gold Coast. Or, il est manifeste qu'en les reléguant, plus encore qu'elles ne l'étaient auparavant, aux tâches domestiques et familiales, ils leur ôtent toute indépendance économique et renforcent leur sujétion face à leur époux. Certes, en tant qu'épouse unique, elles gagnent sans doute quelque pouvoir sur la sphère domestique: mais leur influence se limite strictement à ce domaine. Ceci d'autant plus que l'Église wesleyenne, où elles demeurent minoritaires, notamment aux niveaux les plus élevés, ne constitue guère pour elles un lieu privilégié d'expression et de pouvoir. 


\section{BIBLIOGRAPHIE}

AGBETI, Kofi, 1986, West African Church History, Leiden, Brill, 2 vol.

BAETA, C. G. (ed.), 1968, Christianity in Tropical Africa, London, Oxford University Press.

BERTRAND, Claude-Jean, 1971, Le méthodisme, Paris, Armand Colin.

BIRTWHISTLE, Allen, 1950, Thomas Birch Freeman, West African Pioneer, London, Cargate Press.

BUREAU, R., 1968, « Influences de la christianisation sur les institutions traditionnelles des ethnies côtières du Cameroun » in BAETA, op. cit.

CURTIN, Philip, 1973, The image of Africa : British Ideas and Actions 1780-1850, Madison, University of Wisconsin Press.

DATTA, Ansu \& PORTER, John, 1971, « The Asafo system in historical perspective », in Journal of African History, vol. 12, $\mathrm{n}^{\circ}$ 2, pp. 279-297.

GUTKIND, Peter, 1989, « The canoemen of the Gold Coast », in Cahiers d'Études Africaines, vol. 115-116, n 3-4, pp. 339-376.

HAMMOND, Dorothy, \& JABLOW, A., 1977, The Myth of Africa, New York, Library of Social Sciences. HUGON, Anne, 1995, L'implantation du méthodisme en Côte de l'Or au XIXe siècle. Stratégies d'évangélisation et modalités de diffusion, 1835-1874, Thèse de doctorat de l'Université Paris I, sous la direction de Jean-Pierre Chrétien.

LAWSON, John \& SILVER, Harold, 1973, A Social History of Education in England, London, Methuen.

MACARTHY, Mary, 1983, Social Change and the Growth of British Power in the Gold Coast : the Fante States, 1807-1874, New York and London, University Press of America.

MUDIMBE, V. Y., 1988, The Invention of Africa : Gnosis, Philosophy and the Order of Knowledge, Bloomington and London, Indiana University Press \& James Currey.

PERROT, Dominique, et PREISWERK, Roy, 1975, Ethnocentrisme et histoire, Paris, Anthropos. REYNOLDS, Edward, 1974, Trade and Economic Change on the Gold Coast 1807-1874, London, Longman.

\section{NOTES}

1. Né en Grande-Bretagne au milieu du XVIIIe siècle sous l'impulsion d'un pasteur anglican bientôt dissident, John Wesley, le méthodisme fait partie des Églises non- 
conformistes. Vers 1850, il rassemble environ deux millions de membres et sympathisants en Angleterre, recrutés surtout dans le centre et l'Ouest du pays et dans la lower middle class. La branche missionnaire de cette Église, baptisée Wesleyan Methodist Missionary Society (WMMS), est fondée en 1799 et envoie un premier missionnaire en Côte de l'Or en 1835. Sur le méthodisme, on verra la synthèse en français de Bertrand, 1971.

2. Cette région est essentiellement peuplée par des Fante (entre Elmina et Winneba) et des $\mathrm{Ga}$, groupe culturellement et linguistiquement fort différent, à l'Est de Winneba.

3. Agbeti 1986.

4. Cet article tire son origine d'un doctorat d'université portant sur l'implantation du méthodisme en Côte de l'Or, de 1835, date de l'arrivée du premier missionnaire, à 1874, date de l'annexion de cette région par la Grande-Bretagne (Hugon 1995). L'ambition de ce travail de thèse était d'élaborer une histoire sociale du méthodisme en Côte de l'Or en dehors d'un contexte politique colonial, pour tenter d'expliquer comment et pourquoi cette nouvelle religion avait réussi (ou, le cas échéant, échoué) à s'enraciner. L'essentiel de ce travail s'est fondé sur les archives de la Wesleyan Methodist Missionary Society, qui a récemment légué ses archives (classées) à la School of Oriental and African Studies (Université de Londres). Elles comprennent : la correspondance générale adressée par les missionnaires de Gold Coast à la maison-mère, à Londres (de très loin la série la plus volumineuse); les « minutes synodales », comptes rendus annuels dressant le bilan de la mission de Gold Coast durant l'année écoulée ; les dossiers de candidature (série très incomplète) donnant des indications sur les postulants à un emploi de missionnaire outre-mer ; enfin, la « série spéciale ", qui rassemble divers documents non classés, tels que journaux de missionnaires ou photographies. J'ai pu consulter aussi des documents restés inconnus jusqu'à mon premier séjour au Ghana (mai 1992), registres de baptême remontant à 1838 et contenant des renseignements sur l'âge, la profession et le statut juridique (esclave, libre, ou esclave temporaire) de la personne baptisée ; ainsi que des papiers privés de familles marchandes africaines liées à la mission méthodiste (Archives nationales du Ghana), complétés par plusieurs dossiers du Colonial Office (Archives nationales britanniques, Public Record Office). S'y ajoutent des sources publiées : publications méthodistes (revues missionnaires, comptes rendus annuels et récits de mission), ouvrages ethnographiques, récits de voyage et de séjour, et journaux britanniques tels que le Times.

5. Les dossiers de candidature des aspirants-missionnaires fournissent des renseignements sur la provenance géographique, la profession, l'origine sociale, le niveau d'instruction et le degré de piété des candidats. Grâce à ces documents, on peut dessiner un portrait-robot du missionnaire méthodiste envoyé en Côte de l'Or : issu de la très petite bourgeoisie, souvent des Midlands ou de Cornouailles, doté d'une instruction minimale et d'un bagage culturel réduit, il ne connaît presque rien de l'Afrique.

6. L'expression de " société côtière » se rapporte ici aux sociétés fante et ga de Côte de l'Or, implantées essentiellement le long du littoral et dans une bande de quelques dizaines de kilomètres dans l'arrière-pays.

7. Macarthy 1983, chapitre 1.

8. Datta \& Porter 1971 ; Gutkind 1989. 
9. Rappelons qu'il n'en va pas de même en Europe à la même époque. En France, il faut attendre une loi de 1907 pour qu'une femme mariée puisse disposer à sa guise de son salaire.

10. Pâte de maïs fermenté présentée sous forme de boule, qui constitue la base de l'alimentation des populations côtières.

11. Reynolds 1974.

12. Macarthy $1983: 21$.

13. Le discours européen sur les femmes s'inscrit évidemment dans un contexte plus vaste, celui de l'image de l'Afrique et de ses habitants dans la pensée occidentale au XIXe siècle. Il ne saurait être question de détailler davantage ici, mais on pourra lire à ce sujet Curtin 1964 ; Hammond \& Jablow 1977 ; Mudimbe 1988 ; Perrot \& Preiswerk 1975.

14. WMMS, Freeman, 12 mai 1838.

15. Les réflexions horrifiées des nouveaux-arrivés sur les habitudes vestimentaires des populations africaines relèvent presque du passage obligé dans leurs premières lettres. 16. WMMS, Wrigley, 9 janvier 1837.

17. WMMS, Freeman, compte rendu de 1838.

18. Si les missionnaires du XIXe siècle parlent de " polygamie », le terme de polygynie est préférable dans la mesure où en Gold Coast, seuls les hommes sont autorisés à contracter des mariages multiples simultanés. Les principes énoncés par le Règlement de la WMMS sont très clairs à l'égard de cette institution : "Comme il arrive que certains Nègres vivent en polygames, ou dans un état de promiscuité entre les sexes, vous prendrez soin de condamner et de réprimer ce vice, et de maintenir à ce sujet une stricte discipline dans nos sociétés. Aucun polygame ne saurait être admis comme adhérent, fût-ce à l'essai, s'il ne consent pas à prendre pour épouse l'une de ses femmes à laquelle vous l'unirez par le mariage. » Cette rigueur méthodiste mérite d'être soulignée, car pour être courante parmi les sociétés missionnaires au XIXe siècle, elle n'est pas absolument universelle : la polygynie étant mentionnée dans l'Ancien Testament, certaines missions y voient une raison de ne pas la proscrire formellement. D'ailleurs, en Côte de l'Or, une autre mission protestante, implantée plus au Nord-Est, est connue pour sa tolérance envers la polygynie : les missionnaires de la Mission de Brême admettent le baptême et la communion d'époux polygynes.

19. « La polygamie, qui résulte en une immoralité générale », écrivent les missionnaires dans les minutes synodales de 1874 .

20. WMMS, Journal de Thackwray, 20 février 1841.

21. De façon générale, les raisons invoquées par les femmes qui défendent la polygynie sont d'ordre matériel : elles mettent en avant les avantages qu'il y a à partager le travail domestique et conjugal à plusieurs.

22. WMMS, Journal de Thackwray, 20 février 1841.

23. WMMS, Freeman, compte-rendu de 1838.

24. WMMS, Freeman, compte-rendu de 1838.

25. Le Règlement de la WMMS rejoint en cela le mot d'ordre de Wesley : « Sauver les âmes seulement. » Ce précepte aurait l'avantage de la simplicité, s'il n'était, en pratique, inapplicable sans une action de type laïque sur les sociétés. Les missionnaires de Côte de l'Or en sont d'ailleurs bien conscients, lorsqu'ils écrivent en 1844 : «En raison des circonstances particulières dans lesquelles nous sommes placés dans ce district, nous sommes obligatoirement amenés à effectuer un gros travail séculier, 
interférant parfois douloureusement avec les tâches plus élevées qui devraient être le propre du ministère chrétien. » (WMMS, minutes synodales pour 1843).

26. WMMS, Freeman, compte rendu de 1838.

27. Plusieurs tentatives ont vu le jour, mais aucune ne s'est révélée durable.

28. Prenons pour exemple l'année 1872. Dans le district de Cape Coast, outre une école de filles avec cinquante-deux élèves, on compte cinq écoles « mixtes » dont trois fréquentées par des filles ; pour le district d'Anomabu, les chiffres sont de neuf et six ; pour celui de Domonasi, quatre et trois ; pour celui d'Accra, sept et trois.

29. WMMS, Watson, 13 juillet 1841.

30. WMMS, minutes synodales pour 1848 .

31. Seules les très petites écoles, avec un nombre réduit de fillettes, offrent un enseignement indifférencié.

32. Grammaire, lecture, vocabulaire, orthographe, écriture, géographie et calcul.

33. Minutes synodales pour 1848 . Cette situation rappelle celle de la Grande-Bretagne à la même époque : «L'enseignement de la couture, omniprésent, devient, à partir de 1862, obligatoire si l'on veut toucher des subventions. Il remplace très souvent l'arithmétique. » (Lawson \& Silver $1973: 286$ ).

34. WMMS, Allen, 4 janvier 1843. Ces préoccupations vestimentaires ne s'apppliquent qu'aux filles, puisqu'à la question « Vos élèves sont-ils nourris et habillés par vos soins ? ", le directeur de la mission répondait : « Nous habillons en partie nos élèves dans les écoles de filles. " (WMMS, Freeman, questionnaire de 1841). Précisons que les habits offerts aux écolières par la mission, ainsi que les nombreux nécessaires à ouvrages et coupons de tissu, sont généralement fournis gracieusement par des méthodistes britanniques (WMMS, Allen, 15 octobre 1846). Ces cadeaux sont d'ailleurs utilisés par les missionnaires comme incitation à la scolarisation des filles.

35. WMMS, Freeman, compte rendu de 1838 et supra, note 26.

36. WMMS, Journal de Freeman, 1 mai 1838.

37. Cette citation n'est pas sans rappeler le cas des « écoles de fiancées » du Cameroun, mentionnées par Bureau (in Baëta $1968: 169$ ), puisqu'il s'agit d'établissements destinés à former les épouses des convertis. Cf. aussi Birtwhistle (1950:3) qui écrivait : «Mrs Wrigley ouvrit une école destinée à l'instruction des soins du ménage afin qu'elles deviennent de bonnes épouses pour les enseignants et les prédicateurs à venir. »

38. WMMS, Freeman, compte rendu de 1838.

39. WMMS, Freeman, questionnaire de 1841.

40. WMMS, Freeman, questionnaire de 1841.

41. Les méthodistes s'en rendent d'ailleurs bien compte, puisque le questionnaire de 1841 comporte plusieurs questions relatives à ce problème, telles que : « Les mariages d'indigènes chrétiens s'accompagnent-ils généralement de bonheur domestique ?» (Réponse : "Oui, à de très rares exceptions près »); ou encore : « Les femmes [dans les couples chrétiens] sont-elles souvent abandonnées par leur mari ?» (Réponse :

« Non »). Cf. WMMS, Freeman, compte rendu de 1841.

42. WMMS, journal de Thackwray, 19 mars 1841.

43. Cellule de base de l'Église méthodiste, la classe est constituée de quelques adhérente-s qui se réunissent sous l'égide d'un-e « class-leader ».

44. WMMS, journal de Thackwray, 19 mars 1841.

45. WMMS, minutes synodales pour l'année 1848.

46. Cette limite est sans doute due en partie au fait que les missionnaires sont tous des hommes. 
47. Bertrand $1971: 77$.

48. Un circuit est une division administrative de l'Église méthodiste ; au milieu du XIXe siècle, on compte quatre circuits en Côte de l'Or, tous de taille assez réduite.

49. Soit 1344 femmes sur 3256 baptisés dans le circuit de Cape Coast entre 1838 et 1874 ; et 877 femmes sur 2289 baptisés dans le circuit d'Anomabu entre 1844 et 1874 .

50. Les missionnaires comptabilisent en effet leurs adhérents sans tenir compte du sexe.

51. WMMS, Freeman, compte rendu de 1838 et WMMS, minutes synodales pour l'année 1845.

52. Alors que dès les années 1840, la WMMS recrutait sur place des convertis dotés d'un assez haut niveau d'instruction, engagés avec le titre de missionnaires.

53. A ce titre, il existe des disparités de salaires entre collègues de même sexe recrutés avec le même titre. Et l'on trouve même une maîtresse d'école qui, en 1865, gagne la même somme que son collègue masculin ; mais il s'agit de l'exception qui confirme la règle. En outre, la personne en question, Elizabeth Waldon, jouissait d'un grand renom. D'ascendance euro-africaine, elle avait été élevée dans la meilleure tradition de la bonne société britannique. Pour son éloge funèbre, Freeman affirmait même : « Aucune histoire de la Côte de l'Or ne saurait être considérée comme complète si elle ne mentionne la vie et l'oeuvre de Elizabeth Waldon. » - recommandation passée largement inaperçue depuis...

\section{RÉSUMÉS}

Les missionnaires méthodistes de Gold Coast au XIX ${ }^{\mathrm{e}}$ siècle ont analysé la place des femmes dans la société africaine locale en termes de subordination. Dès lors, ils élaborent un discours qui se veut émancipateur et se proposent d'améliorer le statut et la condition des Africaines grâce à l'instruction ou au mariage monogame. Mais le fossé est patent entre théorie et pratique : leur action tend plutôt à renforcer la sujétion des femmes chrétiennes, reléguées à la sphère domestique; au sein de l'Eglise méthodiste elle-même, les femmes demeurent minoritaires, surtout aux postes de responsabilité.

XIX $^{\text {th }}$ Century Methodist missionaries on the Gold Coast developed an analysis of the women's situation in terms of subordination. Hence their discourse which offered to emancipate African women through education and monogamy. However, the gap between theory and practice is wide : missionary actions tended to reinforce women's subordination as christian women were increasingly confined to the domestic sphere; as to their place in the Church itself, they remained a minority, especially in the highest positions.

\section{AUTEUR}

\section{ANNE HUGON}

Anne HUGON. Maître de conférences à l'Université Grenoble II en histoire contemporaine, travaillant sur les relations entre Europe et Afrique au XIXe siècle, elle a traduit l'ouvrage d'une 
exploratrice britannique en Afrique de l'Ouest (Mary Kingsley : Une Odyssée Africaine, Paris, Phébus, 1992) et publié deux ouvrages sur les explorations européennes en Afrique (L'Afrique des explorateurs, Découvertes, Gallimard, 1991 et 1994). Plus récemment, elle s'est attachée aux phénomènes de changements culturels et religieux en Afrique, et elle a soutenu sa thèse de doctorat en 1995 sur l'implantation d'une mission britannique au Ghana. 